\title{
On the Performance of Wi-Fi, Ad Hoc and WiMAX Handovers with MIPv4 versus MIPv6
}

\author{
Fatma A. Al Emam \\ ECE Dept., Fac. Of Eng., \\ Mansoura University \\ Mansoura, Egypt
}

\author{
Mohamed E. Nasr \\ ECE Dept., Fac. Of Eng., \\ Tanta University \\ Tanta, Egypt
}

\author{
Sherif E. Kishk \\ ECE Dept., Fac. Of Eng., \\ Mansoura University \\ Mansoura, Egypt \\ Cairo, Egypt
}

\begin{abstract}
To make the most of the available data rates, coverage areas and different networks; wireless communication must be used .Vertical and Horizontal handover were explored in Wi-Fi, WiMAX and Ad-Hoc environments. The vertical handover between Wi-Fi and other wireless technologies could be achieved using Mobile IP and (ABC) CONCEPT. This paper is a comparative study between Mobile IPv4 and Mobile IPv6 to find out which of them performs better when it comes to send datagram from the correspondent node to the mobile node. The tests were carried out to measure delay and throughput metrics and maintain session continuity of mobile node while roaming .The tests were done using OPNET Modeler and NS2 (Network Simulator 2). The results showed that Mobile IPv6 performed better than the other systems in all the tests done.
\end{abstract}

Keywords

MIPv4, MIPv6, Handover, ABC, MANEMO.

\section{INTRODUCTION}

Mobile IP is an internet standard protocol that Improve the existing internet protocol (IP) to comply with the rapid movement of mobile nodes. Mobile IP has provided mobility support to nodes that either have direct connections to the internet or multi hops away from a fixed infrastructure. In this protocol a mobile Agent (MA) is a special router that maintains location information for mobile hosts. When a mobile node $(\mathrm{MN})$ moves away from its home network, the MA will tunnel packets for the MN. Tunneled packets are usually handled by the MA (located in MN's home network or home agent (HA)) on the MN's visiting network called foreign agent (FA). As a result, the MN can continue its communication with the rest of the Internet even though it is away from home network. Mobile Agent (MA) should do the work as a gateway for all of the Non Grounded Mobile Routers (MR) in the ad hoc network; this composes the so called Mobile Ad-Hoc Network Mobility (MANEMO) [1].

The IEEE 802.11 standard defines two operational modes commonly referred to as ad hoc and infrastructure. In ad hoc mode MNs communicate on a peer-to-peer basis. This mode is usually visualized for a scenario where a group of MNs Temporarily set up a network to communicate among them. In infrastructure mode all $\mathrm{MN}$ transmissions go through an Access Point (AP) which isn't mobile and form part of a wired network.

To support internetworking (handover) between IEEE 802.11 and IEEE 802.16 modes, switching algorithm on mobile node as proposed in Fig. 1 is a must. This algorithm is mainly affected by user's preference, not only by signal strength. Mobile IP protocol is employed for mobile devices within public network.
Mobile ad-hoc networks (MANETs) or multihop wireless networks can be formed without any preexisting infrastructure (fixed base stations). In MANET networks the node can act as host and router at the same time. In multihop wireless networks (MANETs) not all MN's were directly connected to a fixed infrastructure. On the other hand, mobile IP could support nodes that have direct connection (wired or wireless) to the internet. Due to these features, an extension to mobile IP is a must to support multihop wireless networks [2].

The proposed solution is the use of ad-hoc routing protocol in the MANET part of the hybrid network and the MN's that have no direct connection to foreign agent (FA) must use one of gateway discovery schemes. These gateway discovery schemes are classified into three types: reactive, proactive and hybrid. The MN's must determine a route to the destination for the packets it sends. These MN's must support two protocols (mobile IP and MANET routing protocol) to connect to the internet. This requires complicated MNs but in reality the used MNs have limited storage space and computing power. There are a second solution ,to overcome complexity of MN's, was not to use MANET routing protocols in addition to standard IP routing and extending Mobile IP to all parts of the hybrid network. This method overcomes the limitations encountered before and requires no exchange of routing tables between MN's. MANEMO provides the nested Mobile Routers with the most appropriate route towards the infrastructure.

\section{INFRASTRUCTURE NETWORK AND ROAMING LEVEL}

Wi-Fi Network topologies are classified into two modes Infrastructure Mode, Ad-hoc (Infrastructure less) Mode. Adhoc routing protocols deliver packets between hosts within MANET network. Ad-hoc routing protocols could be classified into table-driven (proactive) or on-demand (reactive). The difference between both types is that proactive protocols maintain up-to-date routing information, unlike reactive routing that creates routes on necessity. On the other hand; Infrastructure mode use the normal routing protocols like RIP.

\section{Levels of Roaming:}

There are two different levels of network mobility, Layer 2 roaming across a single Layer 2 network (All of the APs are on the same subnet) and Layer 3 roaming [3]. Example of layer 3 roaming as $\mathrm{MN}$ roams from Wi-Fi to WiMAX .The mobile node radio automatically turns off from one access point to another as needed. L3 handoff consists of agent discovery and registration. Signaling messages (Router Solicitation (RS), Router Advertisement (RA), Binding Update (BU) and Binding Acknowledgment (BA)) messages are exchanged during agent discovery and registration. As a 
mobile node roams from one IP subnet to another, the client device might need to obtain a valid IP address for the new subnet. Since Mobile IP allows MN to use two IP addresses, this facilitates the delivery of packet to $\mathrm{MN}$ based on its location.

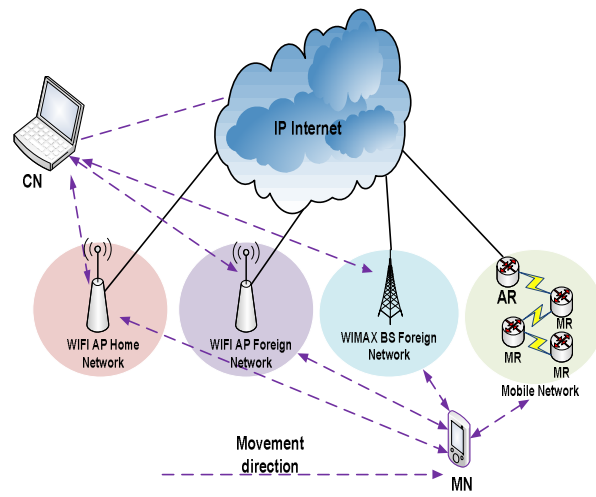

Fig 1: Handover Process

Each MN has a permanent home address (HoA) from its home network. Mobile IP use the concept Care-of-Address (COA) which is the IP of $\mathrm{MN}$ in a foreign network, so the foreign agent (FA) will tunneled and deliver all packets sent to the $\mathrm{MN}$. The foreign agent (FA) must have a connection between it and $\mathrm{MN}$ to be able to deliver packets to the MN. The device that the mobile node (MN) was communicating with is called Correspondent Node $(\mathrm{CN})$. The used technologies are MIPv4, MIPv6; also Fast Handover was discussed that minimize handover delay [4].

MIPv6 features simplify mobility support more than MIPv4 as the foreign agent is not needed anymore, also [5] the $\mathrm{CN}$ can route packets directly to MN. MIPv6 is becoming more popular due to the shortage of globally routable IPv4 addresses so there is no need to use the Network Address Translation (NAT) that causes reachability problems and overcomes the triangular routing problem in MIPV4 where $\mathrm{MN}$ can send packets to $\mathrm{CN}$ through a path to FA. The $\mathrm{CN}$ can deliver packets to MN through HA. This is not sufficient due to the long path between $\mathrm{MN}$ and $\mathrm{CN}$. Tunneling allow two different networks to connect when they were physically disjointed but mobile IP supports Route Optimization (RO) which overcomes drawbacks in Tunneling. Tunneling in mobile IP allows the HA to pass traffic to MN.

Fast Handover for Mobile Ipv6 (FHMIPv6) [6] was proposed by IETF (Internet Engineering Task Force) as extension of MIPv6 to enhance its benefits. Real-Time applications such as VOIP and Video conferencing are affected by handover latency.FHMIPv6 permit MN to connect with new access router more quickly. FHMIPv6 use bi-directional tunnel as proposed in Fig. 2.between Previous Access Router (PAR) and Next Access Router (NAR) to transfer packets during handover. FMIPV6, FHMIPV6 will be discussed later in the next paper.

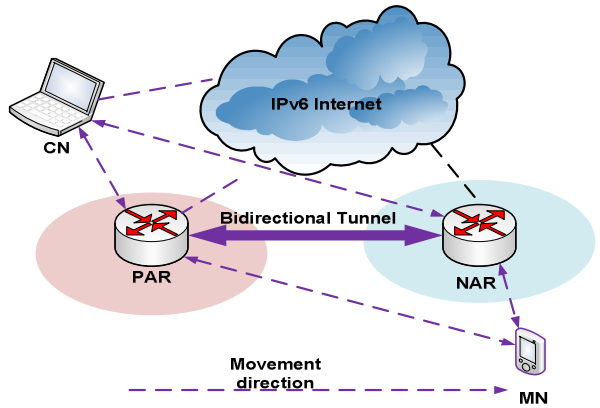

Fig 2: FHMIPv6 algorithm

\subsection{MANEMO NETWORK}

Mobile Ad-Hoc Network Mobility (MANEMO) is integration of MANET and NEMO [7]. This network is suitable for the rescue working environment. A NEMO network consists of a Mobile Router (MR), a Home Agent (HA) and a group of Mobile Nodes (MNs). The MR will manage the mobility of the MNs. If the MNs want to communicate with the MNs in other NEMOs, they should transmit the packets to the MR first, and then the MR will find the routes to the destination. While the NEMO network roaming to a foreign link, the MR will get a Care-of-Address and bind with HA. After the HAMR binding, the HA will receive the packets for the roaming NEMO and transmit them to the MR. So, the NEMO protocol will prevent the loss of connection when a whole network moves from one access point to another, and hence to keep the session continuity. Within the NEMO group, the MNs compose a MANET network and use an Ad-hoc routing protocol to communicate.

\subsection{Vertical Handover Using Abc Concept}

$\mathrm{ABC}$ method is referred to Always Best Connected; means that the nodes are being connected to the best available access technology all the times. The $\mathrm{ABC}$ vision considers multiinterface mobile nodes and multiple simultaneous access technologies in overlapped coverage areas to enable the simultaneous use of different access technologies. The implementation require $\mathrm{Wi}-\mathrm{Fi}$ and WiMAX integrated systems as proposed in Fig. 3. The scenario includes one WiMAX BS, one Wi-Fi AP, two MN's. One MN configured for WLAN and the other configured for WiMAX. Both MN's moved from Wi-Fi AP to Wi-MAX BS.

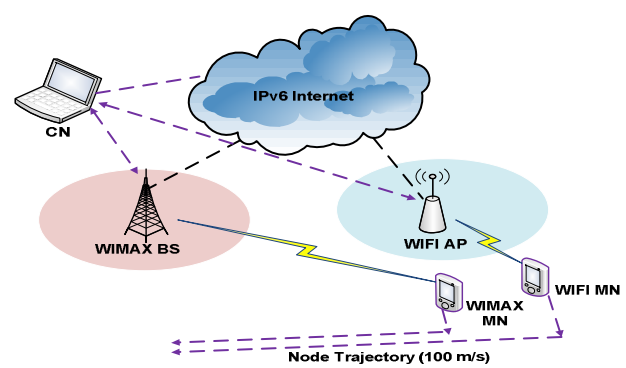

Fig 3: Vertical Handover Using ABC Concept

\section{EXPERIMENTAL DESIGN}

The simulation had been done using Infrastructure Mode MN. This simulation part is divided into three parts: Wi-Fi, WiMAX and MANEMO network; showing different delays and throughput in different wireless networks. All the parts are showing performance behavior in horizontal and vertical handover in Wi-Fi and WiMAX as proposed in Fig. 4. .All the simulation was done using the OPNET 14.5 simulator \& Ns2.33 simulator. 


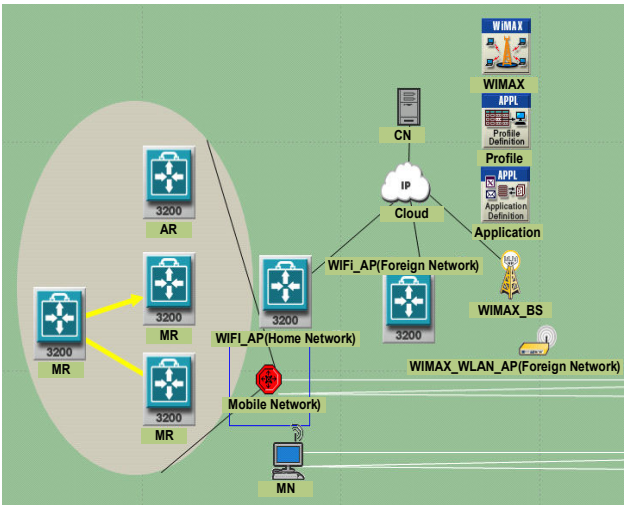

Fig 4: MIPv4 \& MIPv6 Network Structure

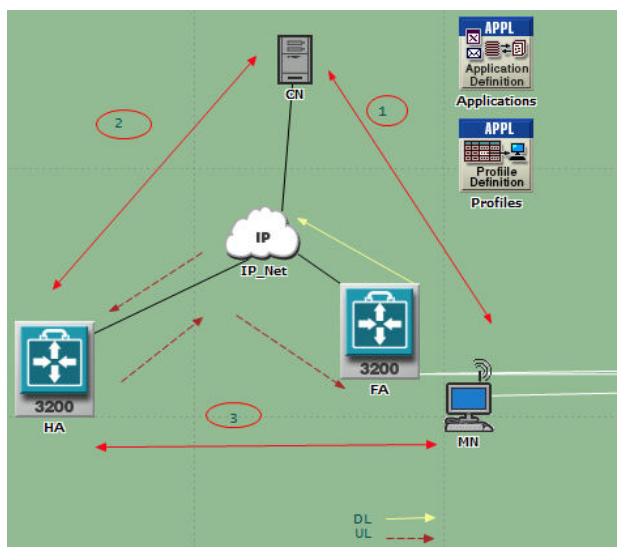

Fig. 5: MIPv4 \& MIPv6 Network Structure.

In MIPv4 scenario, Wi-Fi MN was moving away from HA. Also $\mathrm{MN}$ roaming attribute is enabled. The downlink path (DL) should pass through HA. Unlike Uplink path (UL) should not path through HA. The MN delivers packets destined for the $\mathrm{CN}$ through the FA, which causes triangular routing problem. MIPv6 overcomes this problem. MIPv6 is able to support seamless mobility more efficiently than MIPv4 because of its robustness.MIPv6 also supports route optimization (RO) which results in effective route creation between $\mathrm{MN}$ and the $\mathrm{CN}$.The Uplink path (UL) and downlink path (DL) should not path through HA. The MN and CN could communicate directly. On throughput, RO performed better than BDT. BDT have twice the delay produced by RO.

Each SS_WiMAX_Wi-Fi hot spot (dual-interface router that has Wi-MAX and Wi-Fi interfaces simultaneously) is connected using Wi-MAX connections to Wi-MAX BS. The MN could move from the HA to the FA using mobile IP concept. Here, the delay compared to horizontal handover is larger.

Table 1. Simulation parameters Summary

\begin{tabular}{|c|c|c|c|}
\hline $\begin{array}{c}\text { Coverage } \\
\text { Area }\end{array}$ & $\begin{array}{c}\text { Transmission } \\
\text { power }\end{array}$ & $\begin{array}{l}\text { Data } \\
\text { Rate }\end{array}$ & Application \\
\hline $\begin{array}{c}\text { Square } \\
\text { meters with } \\
1000 \text { meters } \\
\text { length } \\
\end{array}$ & 0.005 & $11 \mathrm{Mbps}$ & $\begin{array}{c}\text { Video } \\
\text { Conferencing } \\
\text {,etc. }\end{array}$ \\
\hline $\begin{array}{l}\text { Ad Hoc } \\
\text { routing } \\
\text { protocol }\end{array}$ & $\begin{array}{c}\text { WLAN routing } \\
\text { protocol }\end{array}$ & $\begin{array}{l}\text { MAC } \\
\text { Type }\end{array}$ & $\begin{array}{c}\text { Reception } \\
\text { power } \\
\text { threshold }\end{array}$ \\
\hline $\begin{array}{l}\text { Ad Hoc on } \\
\text { Demand } \\
\text { Vector } \\
\text { (AODV) }\end{array}$ & $\begin{array}{c}\text { Router } \\
\text { Information } \\
\text { Protocol next } \\
\text { generation } \\
\text { (RIPng) }\end{array}$ & $\begin{array}{c}\text { IEEE } \\
802.11 \\
\text { DCF } \\
\text { IEEE } \\
802.16 \\
\end{array}$ & $-95 \mathrm{dBm}$ \\
\hline
\end{tabular}

\section{SIMULATION RESULTS}

The proposed design has been simulated using the parameters which were given in [8] except the coverage area increased as a result of the use of WiMAX. The below results illustrate the difference between the used techniques.

There is a need to use small data rate to get smooth handoff and decrease the processing on other MN's that transfer packets of other MN's beside its own since in multihop networking the transfer of packets depend on other nodes. Another reason; the larger data rates may be acceptable if movements are infrequent e.g. a mobile node that moves once every 30 minutes. Higher mobility causes more overhead and a need for more processing from intermediate nodes which is not sufficient in our simulation. The main reason behind our simulation is decreasing the delay. The periodic refreshments consume a fraction of the wireless bandwidth as the mobile node requires renewing its COA.

\subsection{WLAN horizontal handover}

TCP tests were carried out in 20 minutes as MN moves from Wi-Fi_AP (home network) to Wi-Fi_AP (foreign network) to measure delay and throughput in MIPv4 and MIPv6. The test results for MIPv4 and MIPv6 are shown below .The results of horizontal handover of WLAN node in infrastructure mode is given in Fig. 6.

MIPv6 suffers a $1.35 \%$ increase in packet size due to its larger IP header [9]. This penalty is the main factor for MIPv6's reduced throughput, while a great improvement, more than $15 \%$, is shown when route optimization is used in the MIPv6 tests. MIPV6 Throughput is larger than MIPV4 due to Route optimization, while delay as we can notice, the time taken for round trip showed that the difference was not so big but MIPv6 was faster than MIPv4.
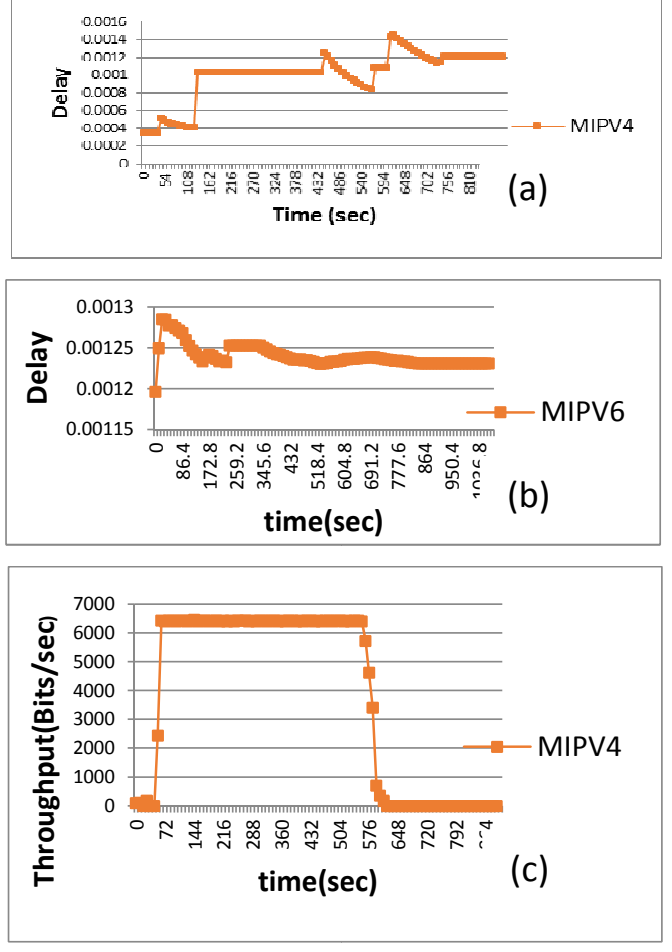


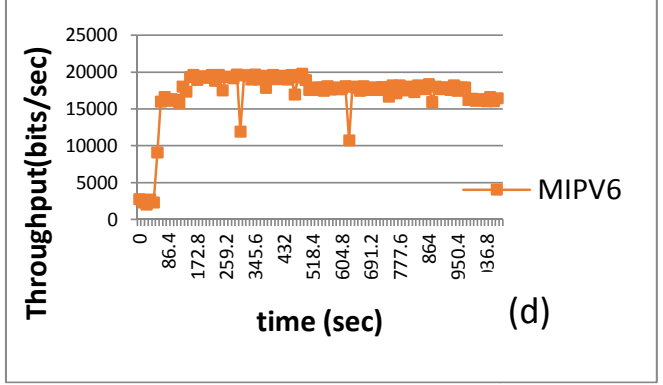

Fig 6: WLAN horizontal handover over MIPv4 \& MIpv6 delay \&throughput respectively.

\subsection{WLAN Vertical handover using ABC concept}

As discussed above in the $\mathrm{ABC}$ [Always Best Connected] concept with LAB components ( section 2.2) .The throughput as proposed in Fig. 7 from WLAN MN initially $=0$ while WiMAX MN throughput have a value. When both MN's start moving towards WiMAX BS then the throughput start increasing and then become constant.[10]

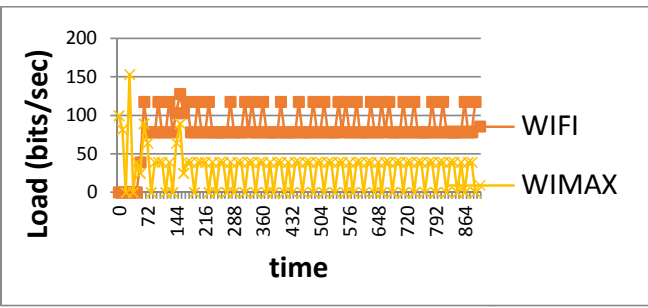

Fig 7: WLAN Vertical handover using ABC concept

\subsection{WLAN vertical handover}

As MN moves from Wi-Fi_AP (home network) to Wi-Fi_AP (foreign network) then to WiMAX_WLAN_AP (foreign network) .This lab slightly differ from the horizontal handover; here as proposed in Fig. 8 (WiMAX_ss_wlan_router) as a dual interface router had been added .The time of transition from Wi-Fi to WiMAX suffers degradation in throughput because of the processing needed at this time at $\mathrm{MN}$ and the delay increases accordingly. After the delay reaches its maximum value it decreases again to reach its normal value as $\mathrm{MN}$ return back to Wi-Fi_AP (foreign network) [11] following its predefined trajectory. The predefined trajectory forces the mobile node to move back and forth through the different points more than once with regard to defined speeds.

In MIPV6 the degradation in throughput from the interval $\mathrm{t}=384(\mathrm{sec})$ to $\mathrm{t}=720(\mathrm{sec})$ as MN transit from WLAN to WiMAX, while the delay increases in the same interval. Handover latency presented by Zimani Chitedze [5] differs in some aspects from the above simulation e.g. the handover between WiMAX, WLAN and Ad hoc networks instead of WMN (wireless Mesh network) and WLAN. Another difference is the use of two types of addressing IPV4 and IPV6; but the simulation parameters are merely the same.
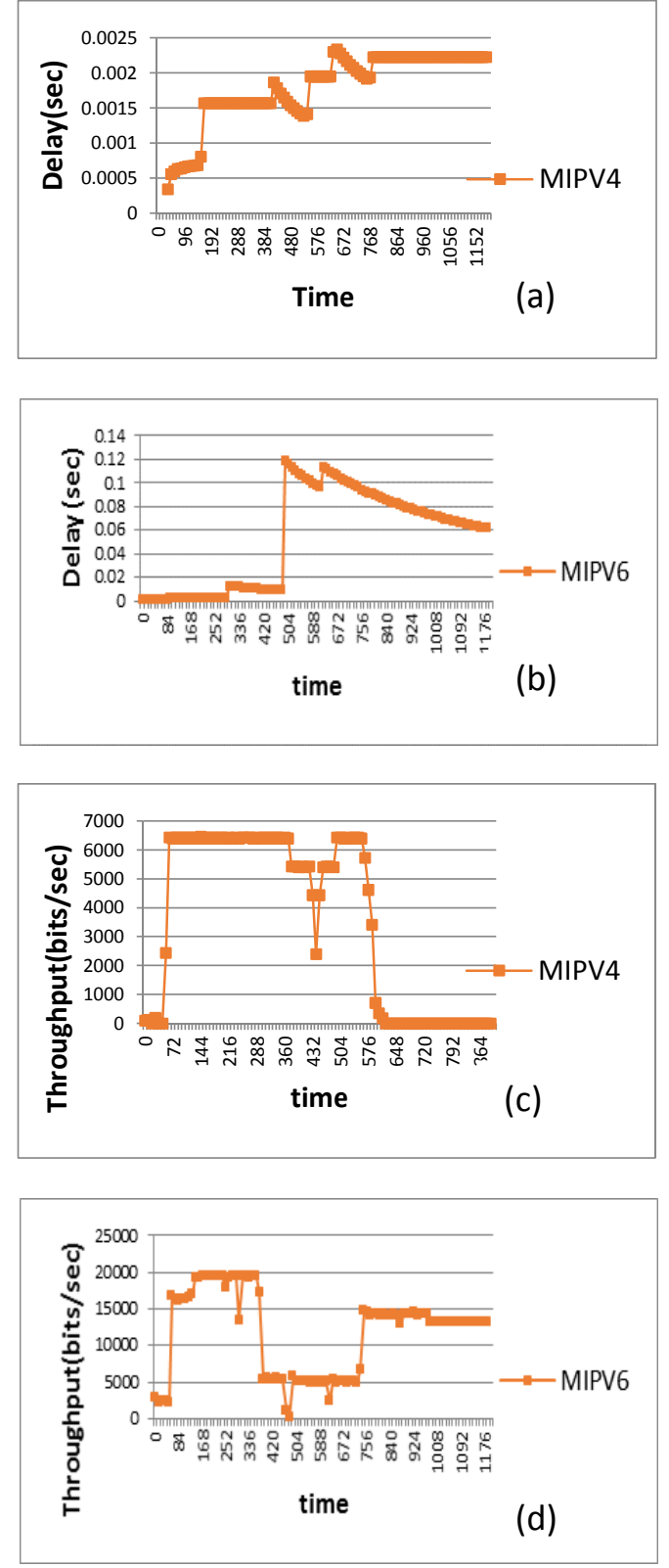

Fig 8: WLAN vertical handover over MIPv4 \& MIpv6 delay \&throughput respectively.

\subsection{MANEMO Horizontal \& Vertical Handover over MIPv4}

horizontal handover occurs as mobile subnet moves from WiFi_AP (home network) to Wi-Fi_AP (foreign network); while vertical handover occurs as the mobile subnet moves towards WiMAX_WLAN_AP (foreign network).Simulation of such scenario was achieved using mobile network that take a predefined trajectory. This mobile network composes of mobile routers that communicate using Ad-Hoc routing protocols with each other. The Movement of mobile network (subnet) from WLAN(home network) moving towards WiMAX (foreign network) appeared as transition points in the delay and throughput curves. The vertical handover begins at $300 \mathrm{sec}$ into the simulation then wait time at WiMAX (foreign network) $1 \mathrm{sec}$ and then return back to WLAN (home network). Since throughput corresponds to the amount of data that is transmitted between $\mathrm{MN}$ and $\mathrm{CN}$ per period of time and Ad-Hoc network different in structure as discussed above; 
the throughput as proposed in Fig. 9 degrades to noticeable value than before and this degradation caused by the multihop path that the packet goes through. Delay (latency) is the time period between the last and new data packets as MN change its position from previous point of attachment to the new point of attachment during handover. Throughput and delay reaches zero in Vertical case transition. With the development of NEMO, MIPV4 leads into added transmission delays; also the vertical case suffers longer delays than horizontal one due to node processing.
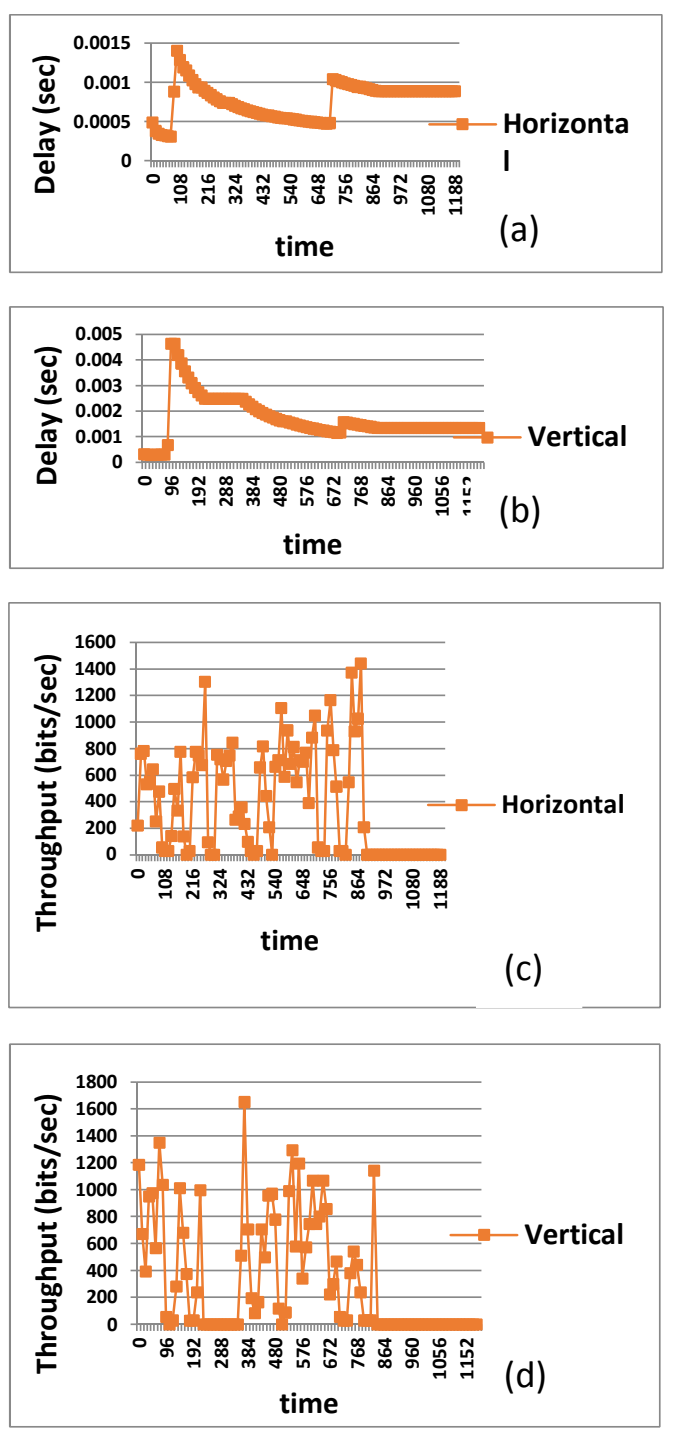

Fig 9: MANEMO Horizontal \& Vertical Handover over MIPv4 delay \&throughput respectively

\subsection{MANEMO Horizontal \& Vertical Handover over MIPv6}

The throughput as said before in MIPV6 is larger than MIPv4 due to route optimization. The throughput as proposed in Fig. 10 is still larger beside the packet in the NEMO network suffers a multihop path to its destination; while delay looks greater especially in vertical handover case of the NEMO network. All trajectories taken in horizontal and vertical are the same.
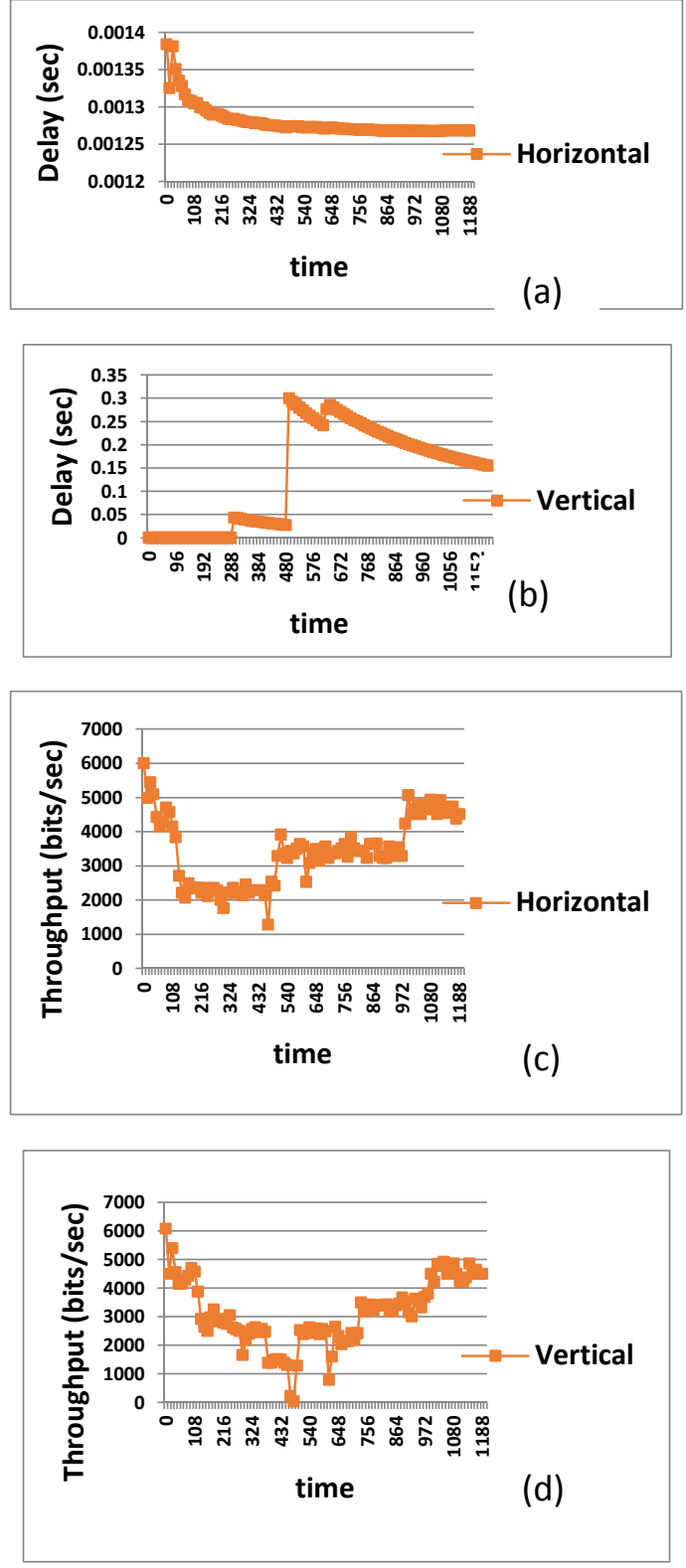

Fig 10: MANEMO Horizontal \& Vertical Handover over MIPv6 delay \&throughput respectively

The following tables show the values of metrics analyzed in the different scenarios like delay and throughput. From the results obtained it can be affirmed that the largest value of throughput was obtained at horizontal handover in MIPV6 with WLAN network marked with gray in Table 2 and the largest value of delay was obtained at vertical handover in MIPV6 with MANEMO network marked with gray in Table 3. Merging all values of the same metric in one table like the below ones Shows clearly the difference between the scenarios in this paper. The points of comparison are HO_Tech (Handover Technique) and Mob Protocol (Mobility Protocol). Fig. 11 is a graphical evaluation of the results as the packet follows its trajectory in the horizontal axis from single hop to multihop network; the delay increase unlike the throughput parameter. 
Table 2. Numerical evaluation of delay parameter

\begin{tabular}{|c|c|c|c|c|}
\hline \multirow{2}{*}{ \%o } & \multicolumn{2}{|c|}{ WLAN } & \multicolumn{2}{c|}{ MANEMO } \\
\cline { 2 - 5 } & MIPV4 & MIPV6 & MIPV4 & MIPV6 \\
\hline Horizontal & 0.0010 & 0.00124 & 0.00140 & 0.00138 \\
\hline vertical & 0.0017 & 0.05313 & 0.00463 & 0.30061 \\
\hline
\end{tabular}

Table 3. Numerical evaluation of throughput parameter

\begin{tabular}{|c|c|c|c|c|}
\hline \multirow{2}{*}{ MO" } & \multicolumn{2}{|c|}{ WLAN } & \multicolumn{2}{|c|}{ MANEMO } \\
\hline & MIPV4 & MIPV6 & MIPV4 & MIPV6 \\
\hline Horizontal & 4357.55 & 17023.4 & 1652.66 & 6085.33 \\
\hline vertical & 3596.4 & 11713.7 & 1442.66 & 6005.33 \\
\hline
\end{tabular}

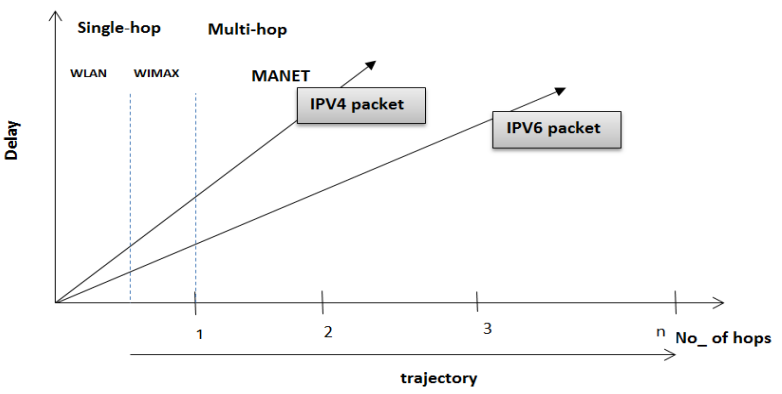

Fig 11: Graphical evaluation of the results

\section{CONCLUSION AND FUTURE WORK}

As a general discussion the factors that affect delay and throughput are the number of foreign agents along the trajectory path of $\mathrm{MN}$ and its location. The delay decrease as the number of foreign agents increase and the distances between them become narrower. The scenario when FA's were placed far away and close to each other were compared. The BDT (Bi-Directional Tunneling) or Route optimization encountered in MIPV6 scenario has reduced delay. The movement speed of MN may be helpful in enhancing performance of handover technique. Another factor that affect delay is Binding Update (BU) which is the mapping of the home address and COA of the MN so that the HA can always recognize the communication of the $\mathrm{MN}$.

In the results MIPV6 is better than MIPV4; though edited version of MIPV6 is needed so as to enhance the measured parameters. These edited versions are (Fast Hierarchal Mobile IP) FHMIP, (Fast Mobile IP) FMIP, (Hierarchal Mobile IP) HMIP. HMIPv6 has better performance than MIPv6 in the wireless domain. FHMIPv6 combines the outstanding features of FMIPv6 and HMIPv6, which could result in even more minimized handover latency and packet loss.

\section{REFERENCES}

[1] Jia Jintao, G. J. "Route Optimization for Nested Mobile Network" http://www.paper.edu.cn/en_releasepaper/cont ent/4457111, 2011,pp. 1-8.

[2] Yu-Chee Tsengy, C.-C. S.-T. "Mobile IP and Ad Hoc Networks: An Integration and Implementation Experience". IEEE Computer,2003, Volume 36, Issue 5, pp. $48-55$

[3] Dhanaraj Cheelu, M. R. "A Study of Vertical Handoff Decision Strategies in Heterogeneous Wireless Networks".International Journal of Engineering and Technology (IJET), 2013,Vol 5 No 3 Jun-Jul 2013 ,pp.2541-2554

[4] Ahmad H. Talaat, N. A."Real-time Traffic Performance for WiFi Handovers over MIPv4 versus MIPv6". International Journal of Computer Applications , July 2011,Volume 26 - No.8 , pp. 10-15

[5] Seneviratne, R. H." A Comparison of Mechanisms for Improving Mobile IP Handoff Latency for End-to-End TCP”. ACM MOBICOM ,Annual International Conference on Mobile Computing and Networking, California, USA, 2003,PP.29-41

[6] Zimani Chitedze, W. D."Mobile Vertical Handover between Wireless LAN and Wireless Mesh Network". Southern Africa Telecommunication Networks and Applications Conference (SATNAC) , September 2011,Volume 2, Issue 6, pp. 212-216

[7] Gordon, J. T. "Delay and Packet Loss Analysis for Handovers between MANETs and NEMO Networks". World Academy of Science\&Engineering and Technology, August 2009,Volume 32, pp. 738-743

[8] Chitedze, Z. Mobility management for Wi-Fi Mobility management for Wi-Fi. University of the Western cape, Faculty of Natural Science, 2012.

[9] Youngjune Gwon, J. K. "Scalability and Robustness Analysis of Mobile IPv6, Fast Mobile IPv6, Hierarchical Mobile IPv6, and Hybrid IPv6 Mobility Protocols Using a Large-scale Simulation". HYPERLINK "http://ieeexplore.ieee.org/xpl/mostRecentIssue.jsp?punu mber $=9179 "$ Communications, 2004 IEEE International Conference on ,2004,Vol 7 , pp.4087-4091

[10] Abhishek Dhiman, K. S. "Vertical and Horizontal Handover in Heterogeneous Wireless Networks using OPNET". International Journal of Engineering Research \& Technology (IJERT) , June 2013,Vol. 2 ,pp. 842-846

[11] Dheyaa Jasim Kadhim, S. S. "Performance and Handoff Evaluation of Heterogeneous Wireless Networks (HWNS) Using OPNET Simulator". International Journal of Electronics and Communication Engineering \& Technology (IJECET), April 2013, Volume 4, Issue 2, pp. $477-496$ 